\title{
Impacto de la Precipitación Anormal en el Crecimiento Económico: El Caso de República Dominicana
}

\author{
EnRiQue Penson*
}

Recibido: octubre, 2018 • Aceptado: diciembre, 2018

Cómo citar: Penson, E. (2019). Impacto de la Precipitación Anormal en el Crecimiento Económico: El Caso de República Dominicana. Ciencia, Economía Y Negocios, 2 (2), 25-44. https://doi.org/10.22206/ceyn.2018.v2i2.pp25-44

\section{Resumen}

En este estudio se estima la relación entre el crecimiento económico y la precipitación anormal, entendida como aquella que no es esperada por agentes económicos que ya incorporan los movimientos estacionales regulares de la precipitación durante el año. Para este propósito se estimaron series indicativas del crecimiento económico provincial de República Dominicana a partir de la evolución de las luces nocturnas en cada provincia, siguiendo la metodología de la literatura internacional. Este crecimiento económico provincial estimado se relacionó con la precipitación anormal a través de un modelo de panel utilizando el método de los momentos generalizado (GMM, por sus siglas en inglés) según la metodología de Arellano \& Bond (1991). Los resultados de las ecuaciones estimadas indican que la precipitación anormal tanto positiva como negativa tiene un efecto de desaceleración en el crecimiento económico, incluso con efectos cuadráticos, lo que significa que mientras más grandes los choques de precipitación, más extremo el efecto en el crecimiento económico. Adicionalmente, se encontró que las provincias más pobres son especialmente vulnerables a los choques de precipitación anormal, y esta relación mediadora es robusta a diferentes medidas de pobreza y/o vulnerabilidad.

Palabras clave: clima; precipitación; crecimiento económico; República Dominicana.

JEL: C33, E32, Q54, Q56

* Analytica Dirección: c/ Boy Scouts No. 16, Santo Domingo, República Dominicana. T: 1-809-885-775. 


\title{
Impact of Abnormal Precipitation on Economic Growth: The Case of the Dominican Republic
}

\author{
ENRIQUe PENSON*
}

Sumitted: september, 2018 • Accepted: december, 2018

\begin{abstract}
This study estimates the relationship between economic growth and abnormal precipitation, that which is not expected by economic agents that already incorporate regular seasonal trends of precipitation $d u$ ring the year. For this purpose series indicative of provincial economic growth in the Dominican Republic were estimated from the evolution of night time lights in each province, following the methodology outlined in the international literature. This estimated provincial economic growth was linked with abnormal precipitation via a panel model using a generalized method of moments (GMM) approach, following the methodology of Arellano \& Bond (1991). The results of the estimated equations indicate that abnormal precipitation, be it positive or negative, has a slowing effect on economic growth, even with quadratic terms, which means that the bigger the precipitation shock, the more extreme the effect on economic growth. Additionally, it was found that the poorest provinces in the country are especially vulnerable to abnormal precipitation shocks, and that this mediating relationship is robust to different measures of poverty and/or vulnerability.
\end{abstract}

Keywords: climate; precipitation; economic growth; Dominican Republic.

* Analytica Address: Street Boy Scouts \#16, Saint Domingue, Dominican Republic. P. $1-809-885-7775$ 


\section{Introducción}

El impacto del clima en el crecimiento económico de economías isleñas pequeñas no es trivial, especialmente en el caso de República Dominicana, que se encuentra en la trayectoria de la mayoría de los fenómenos meteorológicos que atraviesan el Mar Caribe de manera regular. De hecho, República Dominicana ocupa el puesto diez (10) del Índice de Riesgo Climático Global a largo plazo, y se estima que el impacto de solamente las lluvias extremas de noviembre de 2016 y abril de 2017 resultó en pérdidas de alrededor de US\$862 millones al país (Ministerio de Economía, Planificación y Desarrollo, 2018). A pesar de esto, el estudio del impacto del clima en la economía dominicana no ha sido abordado por muchos autores.

El ejercicio de indagar sobre el impacto del clima sobre la economía dominicana se dificulta por al menos tres factores. Primero, no se cuenta con una medición abarcadora de precipitación para todas las provincias de República Dominicana por parte de instituciones locales; segundo, hasta hace poco solo existían estadísticas trimestrales indicativas del crecimiento económico, lo que limita significativamente los grados de libertad de cualquier modelo de series de tiempo a estimarse; y tercero, estas mediciones de crecimiento económico solo se calculan a nivel país, así haciendo imposible aprovechar la heterogeneidad del impacto del clima en las diferentes provincias del país a través de modelos de panel.

Este trabajo encuentra la forma de trabajar y solucionar parcialmente las limitantes mencionadas anteriormente para poder responder las siguientes preguntas: ¿Existe un impacto significativo de la precipitación anormal, entendida como aquella no esperada, en el crecimiento económico provincial? ¿Coinciden las provincias más vulnerables en términos de indicadores de pobreza con aquellas más afectadas por los fenómenos meteorológicos? Estas son algunas de las preguntas que esta investigación trata de responder.

Para responder estas preguntas, y trabajar las limitantes del análisis del impacto del clima en el crecimiento planteadas anteriormente, se propone generar series de crecimiento económico mensual por pro- 
vincia a partir de estimaciones de luminosidad nocturna en las mismas, utilizando coeficientes calibrados con la relación PIB-luminosidad a nivel país; esta metodología se ha usado ampliamente en el plano internacional para generar estimaciones sobre el comportamiento de variables económicas y demográficas en unidades geográficas más finas que las que las series describen originalmente (Donaldson \& Storeygard, 2016). Luego, se generan series de precipitación anormal para cada provincia extrayendo el residuo de regresiones que capturan la estacionalidad regular de la precipitación, así aislando el componente no esperado (choque) en las series. Finalmente, se estiman modelos que relacionan estos crecimientos económicos provinciales con los choques de precipitación anormal.

Los resultados de los modelos que relacionan el crecimiento económico con la precipitación anormal indican que existe una relación negativa entre el crecimiento y choques tanto negativos como positivos de precipitación, incluso con un componente cuadrático, lo que indica que mientras mayores los choques, el efecto negativo en el crecimiento se hace geométricamente más grande. Adicionalmente se comprobó que las provincias más vulnerables son más afectadas por la precipitación anormal, y esta condición es robusta a diversas medidas de vulnerabilidad, como pobreza, hacinamiento, nivel de saneamiento y existencia de necesidades básicas insatisfechas.

El resto de este documento se ordena de la siguiente forma: en la sección 2 se hace un recuento de la literatura internacional y nacional. Luego la sección 3 describe los datos utilizados. Más adelante, en la sección 4, se detalla la metodología de transformación/generación de las series estadísticas a utilizar y formulación de las ecuaciones a estimar. La sección 5 muestra los resultados del proceso de estimación de ecuaciones y la sección 6 presenta las conclusiones del estudio.

\section{Revisión de la literatura}

La siguiente revisión de la literatura relevante para el estudio del efecto de la precipitación anormal en el crecimiento económico se fundamenta en dos bloques principales. Primero, se explora la ex- 
periencia internacional estudiando esta relación, y segundo, se presentan los resultados de los trabajos que han tocado este tema en la República Dominicana.

\section{Literatura Internacional}

Como ha sido notado por autores como Dell, et al. (2014), el estudio del impacto del clima en la actividad económica se enfrenta a una situación ineludible, el clima es, en la mayoría de los casos, fijo; los países cercanos a los polos siempre han sido, y siempre serán, más fríos que los del trópico. Esto significa que es difícil evaluar cómo hubiese sido la economía de República Dominicana si la isla se ubicara en el Mar Báltico (al este de Suecia) y no en el Mar Caribe, y por lo tanto fuese sometida a condiciones climáticas totalmente diferentes. Es por esta razón que los estudios del tipo de corte transversal (analizando la situación en un momento en el tiempo) sufren dificultades al tratar de identificar la relación de causalidad entre el clima y el nivel de actividad económico y/o riqueza de los países.

Una línea relativamente reciente de estudio utiliza modelos de panel (analizando múltiples unidades geográficas a través del tiempo), utilizando ahora los cambios en el clima, y no el nivel, para identificar el impacto de este en la actividad económica. Como es notado por Dell, et al. (2014), el beneficio principal de esta metodología es que sí permite identificar el efecto de variaciones del clima (asumidas exógenas) en diferentes variables económicas.

Esta nueva literatura que aprovecha la metodología de panel para estimar el impacto de los cambios del clima en la actividad económica presenta resultados mixtos en términos de la dirección del choque (positivo o negativo) sobre el crecimiento económico de los cambios del clima. Estudios como Barrios, Bertinelli, and Strobl (2010) han encontrado que mayor precipitación se asocia con mayor crecimiento, mientras que otros como Hsiang (2010) no encuentran efectos de tormentas en el nivel promedio de ingreso de distintos países. Inversamente, Hsiang \& Jina (2014) encuentra evidencia de que las tormentas tienen un efecto negativo en el crecimiento, y no el nivel del 
ingreso, y que el efecto se acumula a través del tiempo; de hecho, el estudio encuentra que el efecto neto de los ciclones durante el período 1970-2008 redujo el ritmo de crecimiento del producto interno bruto (PIB) mundial en $1.3 \%$.

Sobre los aspectos metodológicos, la literatura apunta al uso de modelos que tomen en consideración el sesgo introducido por la inclusión de rezagos de la variable explicada dentro de las ecuaciones de crecimiento estimadas. Aquí revisiones de la literatura como la hecha por Dell, et al. (2014) apuntan a la metodología de Arrelano \& Bond (1991) como una forma viable para eliminar el efecto de este sesgo de la estimación de los coeficientes.

\section{Literatura Dominicana}

La literatura dominicana sobre la relación entre el clima y el crecimiento económico es restringida. Esta relativa escasez se relaciona a las limitantes anteriormente planteadas para este tipo de análisis, como la escasez de datos (tanto de crecimiento como precipitación) con desagregación geográfica dentro del país y una limitada extensión temporal de las series de crecimiento económico.

Recientemente Checo, Mejía y Ramírez (2017) logra estimar modelos explicativos específicamente del producto interno bruto (PIB) del sector agropecuario, encontrando que tanto los choques negativos (sequías) y positivos (exceso de lluvia) de precipitación tienen efectos negativos en el producto del sector. Para encontrar estos resultados utilizaron un indicador que captura el nivel de precipitación del país, calculando el promedio de precipitación de las provincias para las que la Oficina Nacional de Meteorología (ONAMET) posee series de precipitación históricas; luego tomaron este indicador y lo separaron en dos series indicativas de sequías y exceso de precipitación basados en la metodología propuesta por Avella (2001).

Un segundo acercamiento al impacto del clima en la economía fue desarrollado por Ministerio de Economía, Planificación y Desarrollo (2018), que estima las pérdidas causadas por los fenómenos meteorológicos de fechas específicas (noviembre 2016, abril 2017 y septiem- 
bre 2017) a partir del esfuerzo presupuestario que el Estado dominicano tuvo que realizar para restaurar el orden público y los servicios básicos. Este trabajo encuentra que la mayoría de las pérdidas ocurren en las áreas de obras públicas, y agua y alcantarillado. También calcula que las provincias más afectadas, en términos de pérdidas directas, por los fenómenos naturales en el 2017 fueron Puerto Plata, Santiago, Montecristi y Valverde.

\section{Descripción de los datos}

Este trabajo cuenta con dos fuentes de información principales. Primero, se utilizan los archivos digitales del tipo VIIRS suministrados por la Administración Nacional Oceánica y Atmosférica (NOAA, por sus siglas en inglés), que denotan el nivel de luminosidad de las luces nocturnas a través del mundo con un nivel de precisión hasta cuadrículas de 732 metros por 742 metros $(0.55$ $\mathrm{km}^{2}$ ). Los mismos permiten extraer desde su contenido, además de la apariencia visual de las zonas, una variable indicativa del nivel de luminosidad que se ubica entre 0 y $63^{1}$. Estos archivos se encuentran disponibles con frecuencia mensual desde abril de 2012 y abril de 2018 actualmente, por lo que el estudio comprende estas fechas para el análisis. Segundo, se pueden obtener estimaciones del nivel de precipitación mensual por provincia a partir de los datos de la Misión de Medición de Precipitación Tropical (TRMM, por sus siglas en inglés), una iniciativa en conjunto de la NASA y la Agencia de Exploración Aeroespacial de Japón (JAXA). Una tercera fuente utilizada es la serie de IMAE (Indicador Mensual de Actividad Económica) publicada por el Banco Central de la República Dominicana. Finalmente, se extraen una serie de variables indicativas de la pobreza y vulnerabilidad por provincia desde los resultados del Censo de 2010 publicados por la Oficina Nacional de Estadística de la República Dominicana.

\footnotetext{
${ }^{1}$ Ver Elvidge, C., Baugh, K., Zhizhin, M. \& Chi, F. (2013), para una mayor discusión sobre las propiedades técnicas.
} 
Como se puede notar en la tabla debajo, la mayoría de las provincias exhiben valores promedios de luminosidad en los alrededores de 0-2, con marcadas excepciones como Distrito Nacional (39.11) y Santo Domingo (6.27). La mayoría de las series muestra asimetría positiva, indicando que su distribución de frecuencia es más densa en su lado izquierdo que lo esperado de una distribución normal. En el caso de la curtosis de las series, una proporción importante de las mismas muestra valores cercanos a 3 , el valor esperado para una serie con distribución normal.

Tabla 1: Resumen Estadístico de la Luminosidad por Provincia ABRIL 2012 - ABRIL 2018

\begin{tabular}{|c|c|c|c|c|c|c|c|c|}
\hline & Promedio & Std. Dev. & Min. & Max. & Asimetría & Kurtosis & $\rho(1) \rho(12)$ & $\rho(24)$ \\
\hline$\overline{\text { Azua }}$ & 0.32 & 0.11 & 0.07 & 0.58 & 0.49 & 2.94 & $75 \% \quad 20 \%$ & $6 \%$ \\
\hline Bahoruco & 0.34 & 0.14 & 0.07 & 0.72 & 0.45 & 3.06 & $73 \% \quad 37 \%$ & $25 \%$ \\
\hline Barahona & 0.42 & 0.11 & 0.18 & 0.80 & 0.49 & 3.64 & $59 \% \quad 3 \%$ & $7 \%$ \\
\hline Dajabón & 0.22 & 0.12 & -0.03 & 0.56 & 0.74 & 3.13 & $77 \% \quad 24 \%$ & $15 \%$ \\
\hline Distrito Nacional & 39.11 & 3.90 & 28.16 & 49.60 & -0.02 & 3.27 & $38 \% \quad 11 \%$ & $9 \%$ \\
\hline Duarte & 0.73 & 0.19 & 0.40 & 1.16 & 0.56 & 2.63 & $84 \% \quad 27 \%$ & $14 \%$ \\
\hline El Seybo & 0.22 & 0.11 & 0.03 & 0.50 & 0.61 & 3.04 & $72 \% \quad 25 \%$ & $18 \%$ \\
\hline Espaillat & 0.88 & 0.22 & 0.53 & 1.46 & 0.56 & 2.83 & $81 \% \quad 32 \%$ & $12 \%$ \\
\hline Hato Mayor & 0.28 & 0.11 & 0.03 & 0.57 & 0.61 & 3.05 & $73 \% \quad 22 \%$ & $16 \%$ \\
\hline Independencia & 0.18 & 0.11 & -0.05 & 0.45 & 0.47 & 2.57 & $72 \% \quad 22 \%$ & $17 \%$ \\
\hline La Altagracia & 0.75 & 0.18 & 0.46 & 1.31 & 0.97 & 3.53 & $82 \% \quad 33 \%$ & $11 \%$ \\
\hline Elías Piña & 0.17 & 0.11 & -0.08 & 0.45 & 0.61 & 2.90 & $75 \% \quad 22 \%$ & $11 \%$ \\
\hline La Romana & 2.34 & 0.31 & 1.68 & 3.05 & 0.10 & 2.78 & $73 \% \quad 20 \%$ & $21 \%$ \\
\hline La Vega & 0.77 & 0.22 & 0.41 & 1.36 & 0.59 & 2.63 & $81 \% \quad 30 \%$ & $8 \%$ \\
\hline María Trinidad Sánchez & 0.33 & 0.14 & 0.09 & 0.71 & 1.00 & 3.42 & $81 \% \quad 28 \%$ & $6 \%$ \\
\hline Monseñor Nouel & 0.82 & 0.23 & 0.46 & 1.36 & 0.63 & 2.23 & $65 \% \quad 30 \%$ & $-6 \%$ \\
\hline Monte Cristi & 0.31 & 0.15 & 0.04 & 0.71 & 1.01 & 3.07 & $84 \% \quad 22 \%$ & $4 \%$ \\
\hline Monte Plata & 0.23 & 0.12 & -0.04 & 0.53 & 0.73 & 3.12 & $77 \% \quad 21 \%$ & $6 \%$ \\
\hline Pedernales & 0.11 & 0.10 & -0.12 & 0.34 & 0.35 & 2.76 & $68 \% \quad 18 \%$ & $8 \%$ \\
\hline Peravia & 1.07 & 0.24 & 0.58 & 1.62 & 0.12 & 2.36 & $79 \% \quad 21 \%$ & $9 \%$ \\
\hline Puerto Plata & 0.76 & 0.19 & 0.43 & 1.28 & 0.64 & 3.08 & $81 \% \quad 33 \%$ & $17 \%$ \\
\hline Sánchez Ramírez & 0.70 & 0.15 & 0.42 & 1.08 & 0.52 & 2.68 & $72 \% \quad 15 \%$ & $10 \%$ \\
\hline Salcedo & 0.82 & 0.20 & 0.43 & 1.30 & 0.26 & 2.56 & $83 \% \quad 16 \%$ & $11 \%$ \\
\hline Samaná & 0.37 & 0.12 & 0.10 & 0.69 & 0.74 & 3.27 & $72 \% \quad 26 \%$ & $10 \%$ \\
\hline San Cristóbal & 1.69 & 0.24 & 1.24 & 2.30 & 0.23 & 2.51 & $68 \% \quad 21 \%$ & $9 \%$ \\
\hline San José de Ocoa & 0.24 & 0.11 & -0.01 & 0.51 & 0.38 & 3.16 & $71 \% \quad 23 \%$ & $11 \%$ \\
\hline San Juan & 0.38 & 0.11 & 0.15 & 0.64 & 0.42 & 2.69 & $76 \% \quad 20 \%$ & $5 \%$ \\
\hline San Pedro de Macorís & 1.36 & 0.22 & 0.86 & 1.86 & -0.28 & 2.89 & $76 \% \quad 29 \%$ & $15 \%$ \\
\hline Santiago Rodríguez & 0.24 & 0.12 & -0.01 & 0.57 & 0.81 & 3.29 & $79 \% \quad 26 \%$ & $8 \%$ \\
\hline Santiago & 1.60 & 0.28 & 1.02 & 2.38 & 0.36 & 3.04 & $80 \% \quad 25 \%$ & $5 \%$ \\
\hline Santo Domingo & 6.27 & 0.68 & 4.65 & 7.95 & -0.17 & 3.20 & $63 \% \quad 25 \%$ & $18 \%$ \\
\hline Valverde & 0.97 & 0.30 & 0.49 & 1.70 & 0.50 & 2.55 & $90 \% 31 \%$ & $13 \%$ \\
\hline
\end{tabular}

Nota: esta figura muestra el resumen estadístico del nivel de luminosidad por provincia para República Dominicana en el período abril 2012 - abril 2018, construido a partir de datos de la base de datos VIIRS de la NOAA. 
Debajo se muestra el resumen estadístico de la precipitación por provincia. Las provincias con mayor precipitación promedio son Santiago Rodríguez y Samaná, con $150 \mathrm{~mm}^{3}$ y $148 \mathrm{~mm}^{3}$ de lluvia promedio al mes, mientras que en otro extremo están Pedernales, Barahona y Bahoruco, con $71 \mathrm{~mm}^{3}, 74 \mathrm{~mm}^{3}, 89 \mathrm{~mm}^{3}$, respectivamente. La mayoría de las series tienen asimetría positiva, son leptocúrticas (curtosis mayor a 3), y tienen autocorrelación ( $\rho$ ) estable y baja, aún mientras aumenta el número de rezagos.

\section{Tabla 2: Resumen Estadístico de la Precipitación por Provincia}

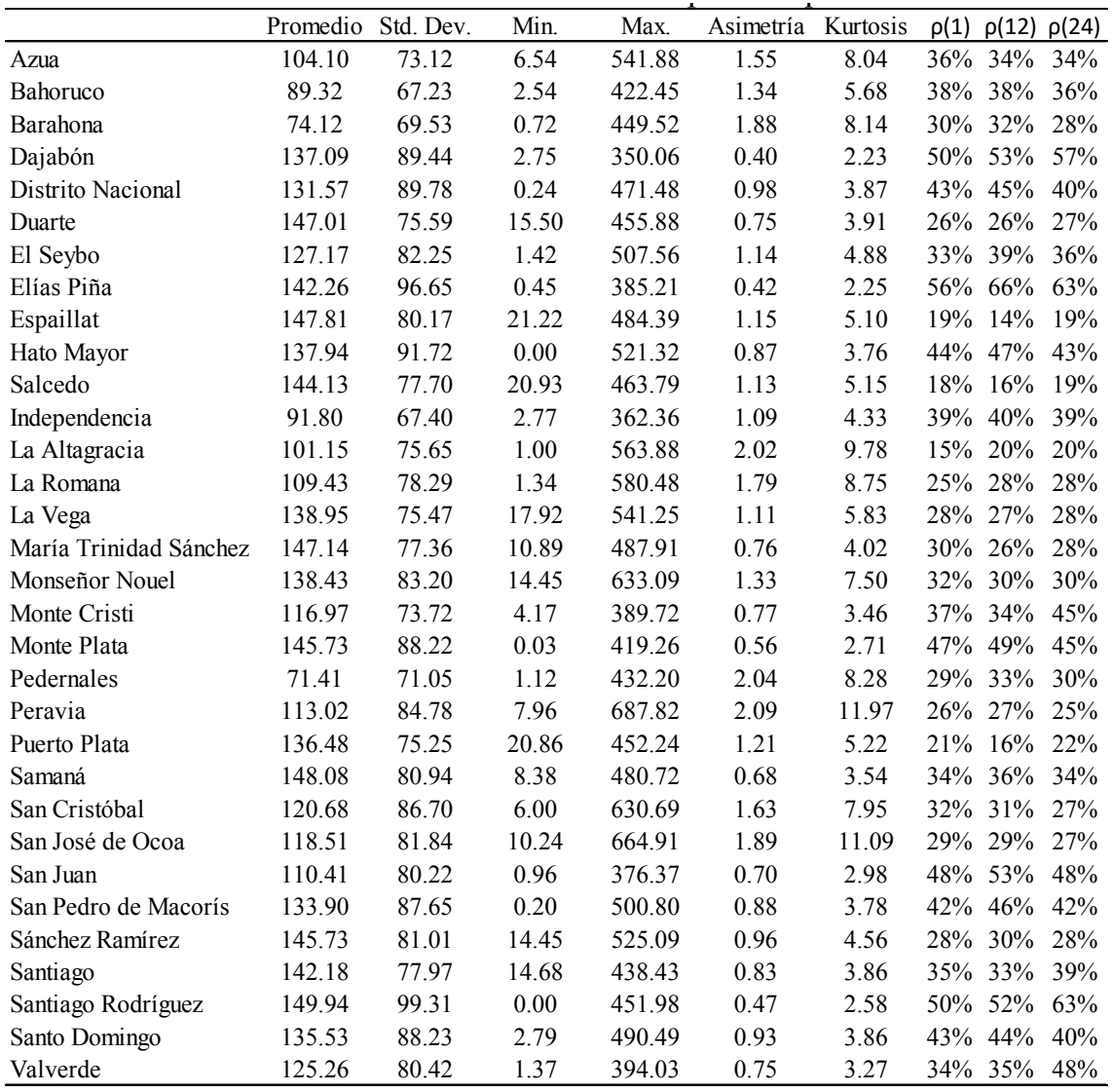

Nota: esta figura muestra el resumen estadístico del nivel de precipitación por provincia para República Dominicana, construido a partir de datos obtenidos desde TRMM.

La siguiente figura muestra la relación entre el nivel del producto interno bruto (PIB) en República Dominicana (medido a través del 
Indicador Mensual de Actividad Económica del Banco Central) con el nivel promedio de luminosidad del país desde abril 2012 a mayo 2018. Se nota una relación positiva entre las variables, interpretando que se esperaría un aumento de $0.47 \%$ en el nivel del producto por cada $1 \%$ que aumente la luminosidad promedio (obtenido a partir de los coeficientes estimados mostrados en la figura). Esta relación lineal captura una parte significativa del comportamiento del producto $\left(\mathrm{R}^{2}\right.$ de $63 \%)$.

\section{Figura 1: Relación entre el IMAE y la Luminosidad para República DOMINICANa}

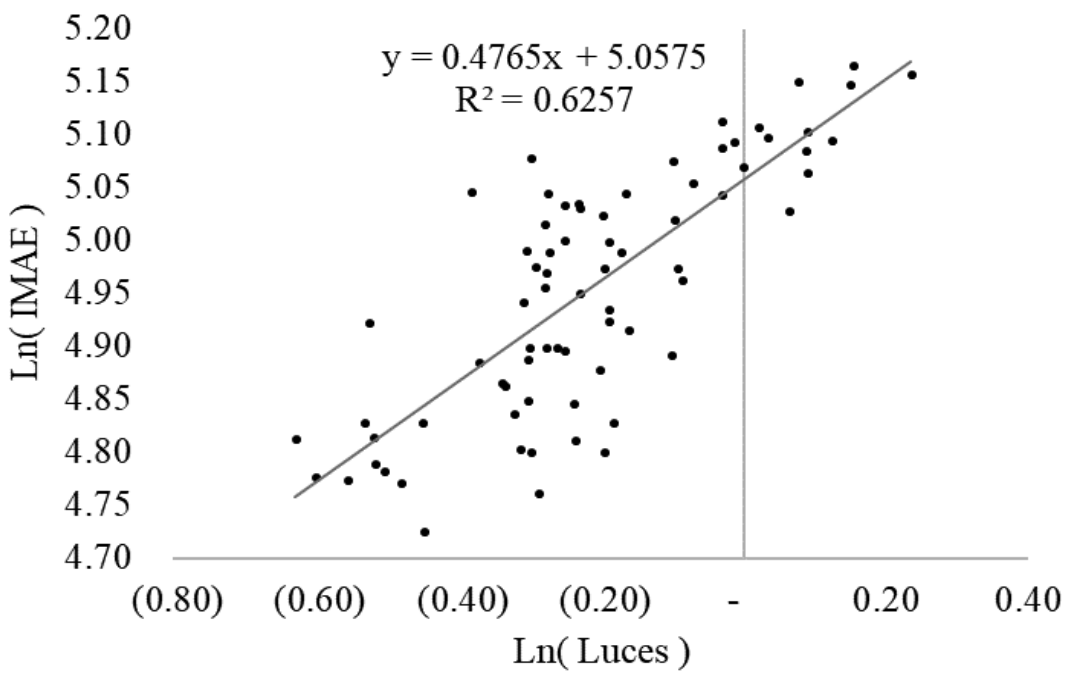

Fuente: IMAE corresponde al Índice Mensual de Actividad Económica publicado de manera mensual, que es indicativo del crecimiento del producto interno bruto (PIB) de República Dominicana. La figura fue construida con datos obtenidos a partir de la Administración Nacional Oceánica y Atmosférica (NOAA, por sus siglas en inglés) y el Banco Central de la República Dominicana. Ambas variables se expresan en logaritmo natural. Se muestra la ecuación estimada de la relación entre las variables con el indicador de bondad de ajuste ( $\mathrm{r}$ cuadrado) debajo. 


\section{Metodología \\ Estimación Crecimiento Provincial}

Habiendo determinado anteriormente la relación estadística entre la luminosidad y el crecimiento económico a nivel país ${ }^{2}$, es posible, de manera similar a la literatura internacional, utilizar estos coeficientes para estimar el crecimiento económico a partir de los cambios en la luminosidad de cada provincia. De esta forma se obtienen series indicativas del nivel de producto de cada provincia, dato que no existe de manera concreta como oferta de los organismos oficiales del Estado Dominicano que publican estadísticas.

Este uso de la luminosidad como variable indicativa (proxy, como se conoce en inglés) del crecimiento económico para unidades geográficas finas (calibrado con las relaciones estimadas a nivel agregado) ha sido explorado ampliamente ya en la literatura por trabajos como Harari (2016), Storeygard (2016), Michalopoulos \& Papaioannou (2013, 2014), Hodler and Raschky (2014), entre otros, como nota Donaldson \& Storeygard (2016).

\section{Obtención de Series de Precipitación Anormal}

Para los propósitos de esta investigación, se utilizó una metodología simple para extraer la precipitación anormal de las series de precipitación por provincia. Para cada serie se estimó un modelo de la forma:

$$
\mathrm{y}_{t}=\alpha+\beta * \mathrm{M}+e_{t}
$$

Donde $\mathrm{y}_{t}$ denota el nivel de precipitación en el período $t, \alpha$ se refiere a una constante estimada, y el vector de coeficientes $\beta$ corresponde a la estacionalidad regular de las series, obtenida al interactuar con la

\footnotetext{
${ }^{2}$ La Figura 1 en la sección de descripción de datos muestra esta relación visualmente y describe la ecuación estimada entre ambas, junto con su bondad de ajuste (R2).
} 
matriz de dummies (variables dicotómicas) $M$, que contiene 11 dummies indicativas de los meses febrero a diciembre. Dada la estructura de estas ecuaciones, la precipitación anormal corresponderá al componente no explicado, capturado por el residuo $e_{t}$.

El resumen estadístico de estos residuos (efectivamente las medidas a utilizarse de precipitación anormal) se encuentra en la tabla debajo. Por la naturaleza de su construcción ${ }^{3}$, el promedio de todos es igual a cero; la desviación estándar oscila alrededor de $55 \mathrm{~mm}^{3}$ de agua, con mínimos alrededor de negativo $100-150 \mathrm{~mm}^{3}$ y máximos entre $250-400 \mathrm{~mm}^{3}$. La asimetría de las series refleja el hecho de que los máximos son más extremos que los mínimos, quizás evidenciando que los choques de precipitación anormal recibidos por las provincias en promedio son de lluvias extremas (República Dominicana es azotada por tormentas tropicales y huracanes con cierta frecuencia), más que de sequías.

La totalidad de las series de precipitación anormal son leptocúrticas (curtosis mayor a 3, que sería la de una serie con distribución normal), indicando que las mismas tienen una distribución con más valores atípicos (extremos) que una distribución normal. Finalmente, como era de esperarse de una serie indicativa de ruido o fenómenos anormales, no se observa mucha inercia, como se observa en las columnas indicativas de la autocorrelación $(\rho)$ a 1, 12 y 24 meses de rezago.

\footnotetext{
${ }^{3}$ Dado que el modelo utilizado para estimar cada residuo contiene un factor constante, este recoge cualquier desviación de cero que la serie presente en promedio, así forzando el valor esperado del residuo a cero.
} 
Tabla 3: Resumen Estadístico de la Precipitación Anormal por Provincia

\begin{tabular}{|c|c|c|c|c|c|c|c|c|c|}
\hline & Promedio & Std. Dev. & Min. & Max. & Asimetría & Kurtosis & $\rho(1)$ & $\rho(12)$ & $\rho(24)$ \\
\hline Azua & 0.00 & 55.16 & -132.96 & 370.65 & 1.76 & 11.56 & $14 \%$ & $-12 \%$ & $-9 \%$ \\
\hline Bahoruco & 0.00 & 49.79 & -109.98 & 268.01 & 1.35 & 7.04 & $17 \%$ & $-10 \%$ & $-9 \%$ \\
\hline Barahona & 0.00 & 54.89 & -108.45 & 300.39 & 1.67 & 8.29 & $10 \%$ & $-6 \%$ & $-10 \%$ \\
\hline Dajabón & 0.00 & 55.05 & -124.87 & 202.40 & 0.51 & 3.66 & $20 \%$ & $-15 \%$ & $5 \%$ \\
\hline Distrito Nacional & 0.00 & 63.61 & -127.45 & 269.64 & 1.08 & 5.04 & $10 \%$ & $-6 \%$ & $-12 \%$ \\
\hline Duarte & 0.00 & 62.66 & -153.24 & 271.05 & 0.78 & 4.80 & $12 \%$ & $-7 \%$ & $-3 \%$ \\
\hline El Seybo & 0.00 & 61.94 & -135.79 & 319.24 & 1.17 & 6.44 & $5 \%$ & $-5 \%$ & $-7 \%$ \\
\hline Elías Piña & 0.00 & 58.45 & -145.74 & 194.91 & 0.43 & 3.59 & $36 \%$ & $15 \%$ & $16 \%$ \\
\hline Espaillat & 0.00 & 69.94 & -159.72 & 279.98 & 0.93 & 5.22 & $15 \%$ & $-13 \%$ & $-3 \%$ \\
\hline Hato Mayor & 0.00 & 63.85 & -139.34 & 310.87 & 0.99 & 5.25 & $10 \%$ & $-6 \%$ & $-9 \%$ \\
\hline Salcedo & 0.00 & 67.41 & -150.28 & 294.72 & 0.98 & 5.49 & $13 \%$ & $-12 \%$ & $-4 \%$ \\
\hline Independencia & 0.00 & 49.17 & -97.84 & 206.64 & 1.08 & 4.97 & $20 \%$ & $-7 \%$ & $-7 \%$ \\
\hline La Altagracia & 0.00 & 63.95 & -126.93 & 409.61 & 1.85 & 10.87 & $-3 \%$ & $-9 \%$ & $-7 \%$ \\
\hline La Romana & 0.00 & 62.55 & -118.26 & 407.88 & 1.81 & 10.85 & $0 \%$ & $-9 \%$ & $-7 \%$ \\
\hline La Vega & 0.00 & 61.26 & -128.70 & 353.09 & 1.14 & 7.46 & $16 \%$ & $-9 \%$ & $-6 \%$ \\
\hline María Trinidad Sánchez & 0.00 & 63.74 & -160.73 & 297.54 & 0.88 & 5.16 & $15 \%$ & $-8 \%$ & $-2 \%$ \\
\hline Monseñor Nouel & 0.00 & 66.31 & -158.39 & 431.30 & 1.47 & 10.14 & $15 \%$ & $-10 \%$ & $-6 \%$ \\
\hline Monte Cristi & 0.00 & 53.77 & -141.15 & 239.14 & 0.90 & 4.99 & $16 \%$ & $-19 \%$ & $8 \%$ \\
\hline Monte Plata & 0.00 & 60.17 & -135.33 & 214.86 & 0.62 & 3.65 & $11 \%$ & $-5 \%$ & $-9 \%$ \\
\hline Pedernales & 0.00 & 56.58 & -117.42 & 276.03 & 1.71 & 7.78 & $10 \%$ & $-3 \%$ & $-5 \%$ \\
\hline Peravia & 0.00 & 69.12 & -160.81 & 497.58 & 2.09 & 14.45 & $7 \%$ & $-9 \%$ & $-9 \%$ \\
\hline Puerto Plata & 0.00 & 64.60 & -154.76 & 259.10 & 1.00 & 5.36 & $16 \%$ & $-14 \%$ & $-1 \%$ \\
\hline Samaná & 0.00 & 63.25 & -159.37 & 296.16 & 0.76 & 4.79 & $13 \%$ & $-3 \%$ & $-3 \%$ \\
\hline San Cristóbal & 0.00 & 68.92 & -147.20 & 435.02 & 1.65 & 9.74 & $10 \%$ & $-7 \%$ & $-11 \%$ \\
\hline San José de Ocoa & 0.00 & 65.38 & -158.48 & 472.93 & 2.03 & 14.50 & $11 \%$ & $-9 \%$ & $-10 \%$ \\
\hline San Juan & 0.00 & 54.38 & -110.94 & 208.94 & 0.85 & 4.47 & $27 \%$ & $4 \%$ & $-2 \%$ \\
\hline San Pedro de Macorís & 0.00 & 61.36 & -128.25 & 298.74 & 1.01 & 5.20 & $7 \%$ & $-7 \%$ & $-9 \%$ \\
\hline Sánchez Ramírez & 0.00 & 65.58 & -141.37 & 333.20 & 0.96 & 5.50 & $13 \%$ & $-6 \%$ & $-7 \%$ \\
\hline Santiago & 0.00 & 59.39 & -135.84 & 261.46 & 0.92 & 5.50 & $20 \%$ & $-13 \%$ & $1 \%$ \\
\hline Santiago Rodríguez & 0.00 & 61.76 & -164.49 & 253.01 & 0.55 & 4.25 & $24 \%$ & $-16 \%$ & $19 \%$ \\
\hline Santo Domingo & 0.00 & 62.94 & -117.77 & 287.89 & 1.01 & 5.01 & $11 \%$ & $-7 \%$ & $-11 \%$ \\
\hline Valverde & 0.00 & 58.37 & -134.95 & 243.71 & 0.86 & 4.76 & $14 \%$ & $-21 \%$ & $11 \%$ \\
\hline
\end{tabular}

Nota: esta figura muestra el resumen estadístico del nivel de precipitación anormal por provincia para República Dominicana, construido a partir de datos obtenidos desde la TRMM.

Debajo se encuentra la tabla de correlaciones cruzadas entre la precipitación anormal de las provincias. La correlación entre la precipitación anormal de las provincias es alta, lo que indica que los choques de precipitación que reciben las provincias son mayormente compartidos entre toda la extensión del país. 


\section{Tabla 4: Correlaciones Cruzadas de la Precipitación Anormal por Provincia}

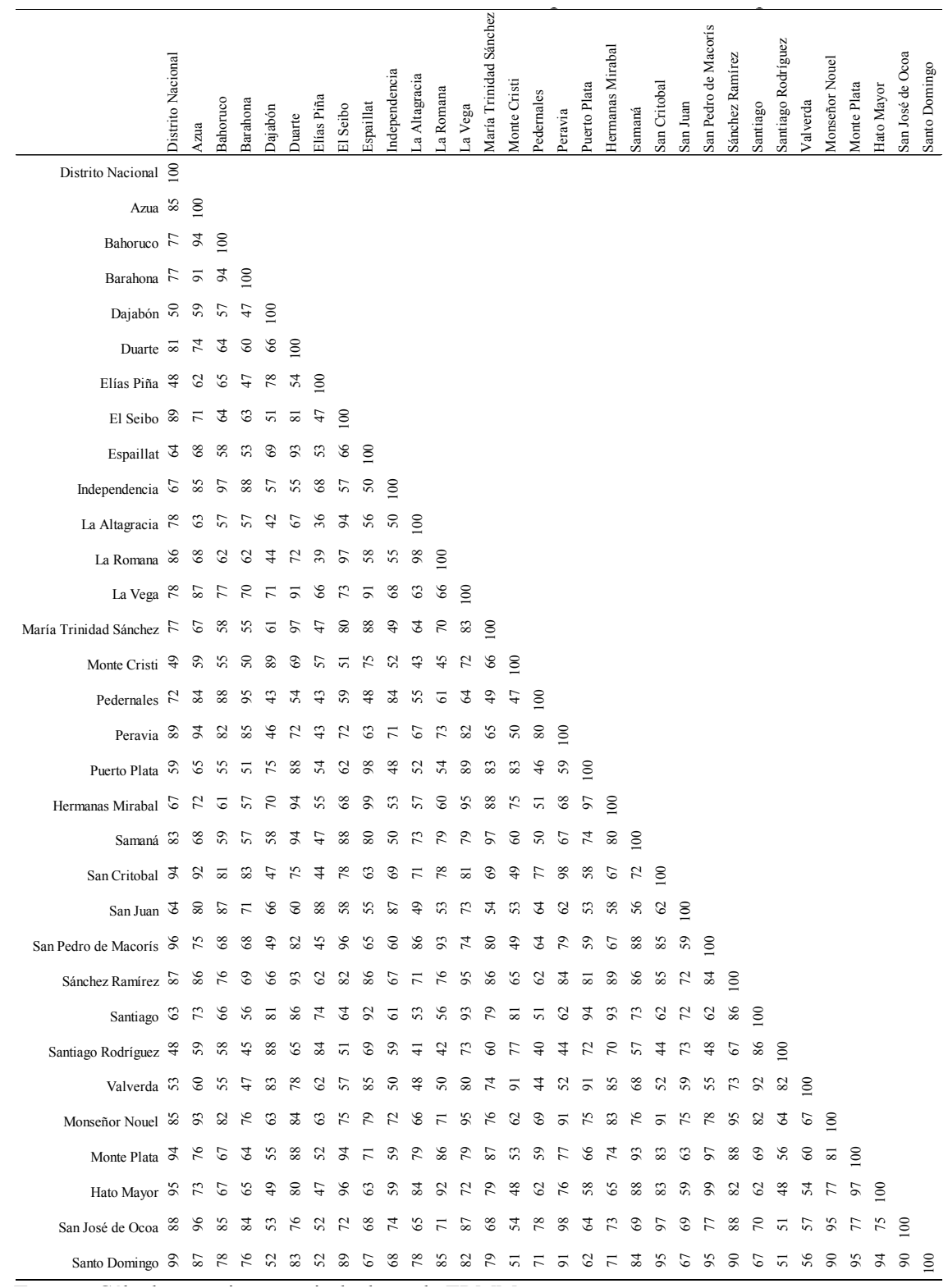

Fuente: Cálculos propios a partir de datos de TRMM. 


\section{Ecuación de crecimiento provincial}

Se propone un modelo del tipo de panel con la siguiente estructura:

$$
\begin{aligned}
\operatorname{dlog}\left(G D P_{i, t}\right) & =\beta_{0}+\beta_{1} * \operatorname{dlog}\left(G D P_{i, t-1}\right)+\theta * M+\varphi_{1} * p c p t_{i, t}^{p} \\
& +\varphi_{2} * p c p t_{i, t-1}^{p}+\varphi_{3} *\left(p c p t_{i, t}^{p}\right)^{2}+\varphi_{4} *\left(p c p t_{i, t-1}^{p}\right)^{2} \\
& +\omega_{1} * p c p t_{i, t}^{n}+\omega_{2} * p c p t_{i, t-1}^{n}+\omega_{3} *\left(p c p t_{i, t}^{n}\right)^{2} \\
& +\omega_{4} *\left(p c p t_{i, t-1}^{n}\right)^{2}+e_{i, t}
\end{aligned}
$$

Donde $d \log \left(G D P_{i, t}\right)$ denota la diferencia logarítmica del producto (PIB) estimado provincial para la provincia $i$ en el período $t$. La matriz $M$ se refiere a un bloque de variables dummies (dicotómicas), de forma que el vector de coeficientes $\theta$ capture la estacionalidad del crecimiento económico. Luego se separó la precipitación anormal de cada provincia en dos variables, siendo $p c p t_{i, t}^{p}$ la precipitación anormal positiva para la provincia $i$ en el período $t, \mathrm{y} \quad p c p t_{i, t}^{n}$ el valor absoluto de la precipitación anormal negativa para la provincia $i$ en el período $t$; de esta forma es posible estimar por separado el efecto en el crecimiento de choques inesperados de precipitación tanto negativos como positivos al crecimiento económico provincial. Adicionalmente, se estimarán una serie de ecuaciones de crecimiento ampliadas que exploren la interacción de variables indicativas de la vulnerabilidad de las provincias con la precipitación anormal.

Debido a la naturaleza dinámica del modelo propuesto, que contiene un rezago de la variable explicada como variable explicativa, los modelos estándares de panel no pueden ser estimados de manera consistente porque por construcción va a existir correlación entre la variable explicada rezagada y el error idiosincrático. Para resolver este problema, Arellano \& Bond (1991) propuso un modelo que utiliza el método generalizado de momentos, que incluye la variable explicada en primera diferencia y utiliza rezagos de esta como instrumentos. 


\section{Resultados}

Los resultados de las ecuaciones de crecimiento económico provincial estimadas se encuentran en la tabla debajo. Aquí se observa, primero, que existe un componente significativo de inercia en el crecimiento provincial, reflejado en el término autorregresivo AR(1) incluido en la ecuación.

Según los resultados de las ecuaciones estimadas, observado en la tabla debajo, sí existe cierta estacionalidad en el crecimiento económico de las provincias, mostrando tendencia a la baja (en comparación con enero, que es el mes que no se introduce y por lo tanto sirve de base) en los meses mayo, junio, julio, noviembre y diciembre.

El bloque de coeficientes relacionados a la precipitación anormal positiva (pcpt_p) muestra un efecto negativo significativo en el componente cuadrático con un mes de rezago. Este resultado hace pensar que el efecto de un fuerte choque de precipitación positiva inesperada toma un mes en hacer efecto, y que el impacto se hace geométricamente más grande mientras mayor es el choque de precipitación.

Como se observa en la tabla debajo para el bloque de coeficientes relacionados a la precipitación anormal negativa (pcpt_n), el efecto es mixto, pero definitivamente contemporáneo (el impacto ocurre en el mismo mes). Para valores pequeños de precipitación anormal negativa, el efecto sobre el crecimiento económico provincial es positivo, pero luego se torna negativo en la medida que el componente cuadrático toma fuerza; aquí ocurre un efecto similar al del choque de precipitación positivo, en que mientras mayor el choque, el impacto en el crecimiento se vuelve geométricamente más grande. 
Tabla 5: Regresiones de Crecimiento Económico Provincial

\begin{tabular}{|c|c|c|c|}
\hline & (I) & (II) & (III) \\
\hline $\operatorname{AR}(1)$ & $\begin{array}{l}-0.087 \\
(4.22)^{* *}\end{array}$ & $\begin{array}{c}-0.130 \\
(6.27)^{* *}\end{array}$ & $\begin{array}{c}-0.128 \\
(6.19)^{* *}\end{array}$ \\
\hline $\mathrm{m} 2$ & & $\begin{array}{l}0.006 \\
(1.39)\end{array}$ & $\begin{array}{c}0.007 \\
(1.69)\end{array}$ \\
\hline m3 & & $\begin{array}{l}0.003 \\
(0.81)\end{array}$ & $\begin{array}{c}0.004 \\
(0.92)\end{array}$ \\
\hline $\mathrm{m} 4$ & & $\begin{array}{l}0.008 \\
(1.92)\end{array}$ & $\begin{array}{c}0.010 \\
(2.28)^{*}\end{array}$ \\
\hline m5 & & $\begin{array}{l}-0.019 \\
(4.30)^{* *}\end{array}$ & $\begin{array}{l}-0.017 \\
(3.64)^{* *}\end{array}$ \\
\hline m6 & & $\begin{array}{c}-0.029 \\
(6.95)^{* *}\end{array}$ & $\begin{array}{c}-0.028 \\
(6.70)^{* *}\end{array}$ \\
\hline $\mathrm{m} 7$ & & $\begin{array}{c}-0.015 \\
(3.64)^{* *}\end{array}$ & $\begin{array}{c}-0.014 \\
(3.29)^{* *}\end{array}$ \\
\hline $\mathrm{m} 8$ & & $\begin{array}{l}-0.008 \\
(1.83)\end{array}$ & $\begin{array}{l}-0.006 \\
(1.53)\end{array}$ \\
\hline $\mathrm{m} 9$ & & $\begin{array}{l}-0.006 \\
(1.47)\end{array}$ & $\begin{array}{l}-0.005 \\
(1.11)\end{array}$ \\
\hline $\mathrm{m} 10$ & & $\begin{array}{l}-0.006 \\
(1.50)\end{array}$ & $\begin{array}{l}-0.004 \\
(1.01)\end{array}$ \\
\hline $\mathrm{mll}$ & & $\begin{array}{c}-0.041 \\
(9.82)^{* *}\end{array}$ & $\begin{array}{l}-0.035 \\
(7.67)^{* *}\end{array}$ \\
\hline $\mathrm{m} 12$ & & $\begin{array}{l}-0.018 \\
(4.21)^{* *}\end{array}$ & $\begin{array}{l}-0.014 \\
(3.01)^{* *}\end{array}$ \\
\hline pcpt_p & & & $\begin{array}{c}0.004 \\
(0.60)\end{array}$ \\
\hline L.pcpt_p & & & $\begin{array}{l}0.005 \\
(0.73)\end{array}$ \\
\hline pept_p_sqr & & & $\begin{array}{l}-0.002 \\
(0.72)\end{array}$ \\
\hline L.pcpt_p_sqr & & & $\begin{array}{l}-0.007 \\
(2.21)^{*}\end{array}$ \\
\hline pcpt n & & & $\begin{array}{c}0.022 \\
(2.51)^{*}\end{array}$ \\
\hline L.pcpt_n & & & $\begin{array}{l}0.005 \\
(0.58)\end{array}$ \\
\hline pcpt_n_sqr & & & $\begin{array}{l}-0.024 \\
(3.13)^{* *}\end{array}$ \\
\hline L.pcpt_n_sqr & & & $\begin{array}{l}-0.006 \\
(0.85) \\
\end{array}$ \\
\hline Constante & $\begin{array}{l}0.001 \\
(1.41) \\
\end{array}$ & $\begin{array}{c}0.011 \\
(3.88)^{* *}\end{array}$ & $\begin{array}{l}0.008 \\
(2.10)^{*} \\
\end{array}$ \\
\hline$N$ & 2,190 & 2,190 & 2,190 \\
\hline
\end{tabular}

Nota: Los asteriscos mostrando el nivel de significancia estadística asociado; “*” denota significancia al 95\% de confianza y “**” al 99\%). Debajo de los coeficientes estimados se encuentra el estadístico t. Debajo se muestra el número de observaciones $(\mathrm{N})$. Las variables m2-12 se refieren a las dummies mensuales. El prefijo "L." se refiere al primer rezago. "pcpt_p" denota la precipitación anormal positiva y "pcpt_n" la negativa. El sufijo “_sqr" se refiere a la transformación cuadrática de una variable. Las variables de precipitación se introdujeron divididas entre 100. 
Como se nota en la tabla debajo, al ampliar la ecuación de crecimiento económico provincial incluyendo interacciones de algunos indicadores representativos de vulnerabilidad (grupos socioeconómicos bajos, hacinamiento, bajo saneamiento y necesidades básicas insatisfechas), se nota que las provincias cuya vulnerabilidad es más alta son más sensibles a choques de precipitación anormal. Esto se evidencia en el signo negativo de los coeficientes de las interacciones en los términos cuadráticos interactuados.

Tabla 6: Regresiones de Crecimiento Económico Provincial Ampliadas

\begin{tabular}{lcccccc}
\hline & $(\mathrm{I})$ & $(\mathrm{II})$ & $(\mathrm{III})$ & $(\mathrm{IV})$ & $(\mathrm{V})$ & (VI) \\
\hline AR(1) & -0.128 & -0.127 & -0.127 & -0.127 & -0.127 & -0.127 \\
& $(6.19)^{* *}$ & $(6.20)^{* *}$ & $(6.18)^{* *}$ & $(6.13)^{* *}$ & $(6.13)^{* *}$ & $(6.14)^{* *}$ \\
Dummies Mensuales & Sí & Sí & Sí & Sí & Sí & Sí \\
& & & & & & \\
L.pcpt_p_sqr & -0.007 & 0.007 & 0.025 & 0.001 & 0.018 & 0.027 \\
& $(2.21)^{*}$ & $(1.70)$ & $(4.37)^{* *}$ & $(0.25)$ & $(1.84)$ & $(2.90)^{* *}$ \\
pcpt_n & 0.022 & 0.013 & 0.005 & 0.002 & -0.009 & 0.003 \\
& $(2.51)^{*}$ & $(0.91)$ & $(0.21)$ & $(0.10)$ & $(0.19)$ & $(0.07)$ \\
pcpt_n_sqr & -0.024 & -0.002 & 0.018 & 0.007 & 0.035 & 0.023 \\
& $(3.13)^{* *}$ & $(0.16)$ & $(0.87)$ & $(0.35)$ & $(0.77)$ & $(0.66)$ \\
L.pcpt_p_sqr (\#) & & -0.001 & -0.001 & -0.000 & -0.001 & -0.000 \\
& & $(6.52)^{* *}$ & $(6.94)^{* *}$ & $(2.53)^{*}$ & $(2.74)^{* *}$ & $(3.93)^{* *}$ \\
pcpt_n (\#) & & 0.001 & 0.000 & 0.001 & 0.001 & 0.000 \\
& & $(0.97)$ & $(0.85)$ & $(1.19)$ & $(0.68)$ & $(0.56)$ \\
pcpt_n_sqr (\#) & & -0.002 & -0.001 & -0.002 & -0.002 & -0.001 \\
& & $(2.30)^{*}$ & $(2.20)^{*}$ & $(1.75)$ & $(1.31)$ & $(1.38)$ \\
cons & 0.008 & 0.008 & 0.007 & 0.008 & 0.008 \\
Interacción (\#) & $(2.10)^{*}$ & $(2.11)^{*}$ & $(2.19)^{*}$ & $(1.99)^{*}$ & $(2.15)^{*}$ & $(2.17)^{*}$ \\
\hline$N$ & & GSE_B_MB & GSE_MB & Hacinamiento & NBI & Saneamiento \\
\hline
\end{tabular}

Nota: Cada modelo cuenta con el coeficiente asociado a la variable, con asteriscos mostrando el nivel de significancia estadística asociado; “*” denota significancia al 95\% de confianza y “**" al 99\%). Debajo de los coeficientes estimados se encuentra el estadístico t. Debajo se muestra el número de observaciones $(\mathrm{N})$. Se indica la inclusión o no de dummies mensuales en los modelos. El prefijo "L." se refiere al primer rezago. "pcpt_p" denota la precipitación anormal positiva y "pcpt_n” la negativa. El sufijo "_sqr" se refiere a la transformación cuadrática de una variable. Las variables con el símbolo "(\#)" a la derecha indican interacciones de las mismas con la variable descrita debajo en la fila de "Interacción (\#)". GSE_B_MB se refiere a grupo socioeconómico muy bajo y bajo, GSE_MB se refiere al grupo socioeconómico muy bajo, Hacinamiento al porcentaje de hogares en condiciones de hacinamiento, NBI al porcentaje de hogares con necesidades básicas insatisfechas y Saneamiento al porcentaje de hogares con bajo y medio saneamiento. Las variables de precipitación se introdujeron divididas entre 100. 


\section{Conclusión}

En esta investigación se comprueba el impacto negativo de los choques de precipitación anormal en el crecimiento económico de las provincias de República Dominicana. Las estimaciones revelan que tanto los choques positivos y negativos de precipitación anormal tienen un efecto negativo y significativo, incluso aumentando geométricamente según la magnitud del choque. Adicionalmente, se comprueba que las provincias más vulnerables son las más afectadas por los choques de precipitación anormal, y este hallazgo es robusto a diferentes medidas de vulnerabilidad.

\section{Referencias bibliográficas}

Arellano, M., \& S. Bond. (1991). Some tests of specification for panel data: Monte Carlo evidence and an application to employment equations. Review of Economic Studies 58, 277-297. DOI:10.2307/2297968

Avella, R. (2001). "Efecto de las Sequías sobre la Inflación en Colombia” Borradores de Economía 183. Banco de la República de Colombia.

Barrios, S., Bertinelli, L., \& Stobl, E. (2010). Trends in Rainfall and Economic Growth in Africa: A Neglected Cause of the African Growth Tragedy. Review of Economics and Statistics, 92(2), 350-66. DOI:10.1162/rest.2010.11212

Checo, A. M., Mejía, M. \& Ramírez, F. A. (2017). El Rol de los Regímenes de Precipitaciones sobre la Dinámica de Precios y Actividad del Sector Agropecuario de la República Dominicana durante el período 2000-2016. Documento de Trabajo 2017-01. Banco Central de la República Dominicana.

Donaldson, D. \& Storeygard, A. (2016). The View from Above, Applications of Satellite Data in Economics. Journal of Economic Perspectives, 30(4), 171-198. DOI:10.1257/jep.30.4.171

Dell, M., Jones, B. F., Olken, B. A. (2014). What Do We Learn from 
the Weather? The New Climate-Economy Literature. Journal of Economic Literature, 52(3), 740-798. DOI:10.1257/ jel.52.3.740

Elvidge, C., Baugh, K., Zhizhin, M. \& Chi, F. (2013). Why VIRRS data are superior to DMSP for mapping nighttime lights. Proceedings of the Asia-Pacific Advanced Network, 35, 62-69. DOI:10.7125/APAN.35.7

Harari, M. (2016). "Cities in Bad Shape; Urban Geometry in India." February. http://real.wharton.upenn.edu/ harari/Harari_Papers/CityShapeHarariMarch2016_updated.pdf.

Hodler, R. \& Raschky, P. A. (2014). Regional Favoritism. Quarterly Journal of Economics, 129(2), 995-1033. DOI:10.1093/qje/ qju004

Hsiang, S. M. (2010). Temperatures and Cyclones Strongly Associated with Economic Production in the Caribbean and Central America. Proceedings of the National Academy of Sciences 107(35), 15367-15372. DOI:10.1073/pnas.1009510107

Hsiang, Solomon M. and Amir Jina. (2014). The Causal Effect of Environmental Catastrophe on Long-Run Economic Growth: Evidence From 6,700 Cyclones. NBER Working Paper No. 20352. DOI:10.3386/w20352

Michalopoulos, S. \& Papaioannou, E. (2013). Pre-colonial Ethnic Institutions and Contemporary African Development. Econometrica, 81(1): 113-52. DOI:10.3982/ECTA9613

Michalopoulos, S. \& Papaioannou E. (2014). National Institutions and Subnational Development in Africa. Quarterly Journal of Economics, 129(1), 151-213. DOI:10.3386/w18275

Ministerio de Economía, Planificación y Desarrollo. (2018). Análisis del Desempeño Económico y Social de República Dominicana 2017.

Storeygard, A. (2016). Farther on Down the Road: Transport Costs, Trade and Urban Growth in Sub-Saharan Africa. Review of Economic Studies, 83(3): 1263-95. DOI:10.1093/restud/rdw020 